\title{
Utilización de una biopelícula microalgal multiespecífica para optimizar la fijación larval y el crecimiento de abalón (Haliotis rufescens) en un criadero comercial
}

\author{
Application of multi-specific microalgae biofilm for optimization of the larvae settlement \\ and growth of abalone (Haliotis rufescens) in a commercial hatchery \\ Rubén Araya $^{1}$, Claudia Bahamondes ${ }^{2}$, Karina Barahona $^{2}$ y Fernando Silva-Aciares ${ }^{2}$ \\ ${ }^{1}$ Laboratorio de Microbiología de Sedimentos, Departamento de Acuicultura, Facultad de Recursos del Mar, \\ Universidad de Antofagasta, Casilla 170, Antofagasta, Chile \\ ${ }^{2}$ Laboratorio de Ecología Microbiana, Departamento de Acuicultura, Facultad de Recursos del Mar, \\ Universidad de Antofagasta, Casilla 170, Antofagasta, Chile. \\ raraya@uantof.cl
}

\begin{abstract}
This study evaluates the use of a microalgal biofilm composed by a mixture of benthic diatoms such as Amphora cf. capitellata (NV), Nitzschia ovalis arnott (NC), Cylindrotheca closterium (CC) and Navicula cryptocephala (NAV) to improve larval settlement and growth of $H$. rufescens postlarvae in commercial hatcheries in the north of Chile. Preliminarily the optimal growth dynamic conditions of selected diatoms were evaluated in relation light intensity by the use of polycarbonate carboys containing PVC filaments for supporting the growth of diatoms. The results showed that the different diatom strains reached the highest cell densities in terms of cell coverage, at $50 \mu \mathrm{mol} \cdot \mathrm{m}^{-2} \cdot \mathrm{s}^{-1}$ of light intensity after $120 \mathrm{~h}$ for NC, CC and NV strains and $144 \mathrm{~h}$ for NAV strain. These results guarantee the massive productions of the selected diatoms for their use as commercial scale. The determination of the multi-especific effect of selected diatoms on the settlement and growth of differents postlarvae cohorts, after 60 and 120 days from the larval metamorphosis, showed significant improvements on settlement and growth of abalone seeds. These results suggest that the utilization of benthic diatom mixtures for the culture of abalone postlarvae at commercial scale improve the disponibility of food for the abalone Chilean industry.
\end{abstract}

Key words: Diatoms, settlement, growth
Resumen.- Este estudio evalúa el uso de una biopelícula microalgal compuesta por una mezcla de diatomeas bentónicas como Amphora cf. capitellata (NV), Nitzschia ovalis arnott (NC), Cylindrotheca closterium (CC) y Navicula cryptocephala (NAV), para mejorar el asentamiento y crecimiento de postlarvas de $H$. rufescens en criaderos en el norte de Chile. En forma preliminar se evaluaron las condiciones óptimas de crecimiento de las diatomeas seleccionadas, en relación a la dinámica de crecimiento y la intensidad lumínica óptima, mediante la utilización de botellones de policarbonato con filamentos de PVC como sustrato para el crecimiento bentónico. Los resultados mostraron que las distintas cepas alcanzaron los mejores crecimientos, en términos de cobertura celular, a $50 \mu \mathrm{mol} \mathrm{m} \mathrm{m}^{-2} \mathrm{~s}^{-1}$ de intensidad lumínica, transcurridas $120 \mathrm{~h}$ de cultivo para las cepas NC, CC y NV y 144 h para la cepa NAV, garantizando la producción masiva de estas especies para su uso a nivel comercial. La determinación de esta mezcla multiespecífica de diatomeas sobre el asentamiento y crecimiento de post larvas para distintas cohortes, posterior a 60 y 120 días desde la metamorfosis larval, mostró mejoras significativas sobre el asentamiento y el crecimiento de las semillas de abalón. Estos resultados sugieren que el uso de mezclas de diatomeas bentónicas para el cultivo de postlarvas de abalón a nivel masivo, mejora la oferta alimentaria para la industria abalonera en Chile.

Palabras clave: Diatomeas, asentamiento, crecimiento

\section{Introducción}

En los últimos años, la industria del abalón en Chile se ha desarrollado de manera vertiginosa y desde los inicios de su cultivo en forma comercial, hace no más de 20 años, ya son más de 20 empresas dedicadas a la producción de este recurso. Es así, que la industria abalonera chilena proyecta producir $800 \mathrm{t}$ para el año
2010 (Viana 2006), lo que en términos de volumen equivale a la producción conjunta de países como México, Estados Unidos, Australia y Sudáfrica. Sin embargo, para lograr tal producción, la industria abalonera nacional deberá mejorar algunas debilidades en la producción de semillas, específicamente respecto de la calidad de estas en función de la oferta alimentaria de diatomeas bentónicas. 
Una de las etapas claves en el éxito del cultivo de este recurso se refiere a la etapa posterior al asentamiento postlarval. En esta transición la larva vitelogénica de abalón está exhausta, por lo que debería alimentarse tan pronto sufre la metamorfosis (Bryan \& Qian 1998). En este período, las diatomeas bentónicas son la principal fuente alimenticia para postlarvas en criaderos (Kawamura et al. 1998, Gordon et al. 2006), constituyéndose estas como la dieta inicial de postlarvas a partir de biopelículas desarrolladas en forma natural sobre los sistemas de cultivos utilizados (Daume et al. 2000, Searcy-Bernal et al. 2001).

A pesar de que los estudios relacionados a la nutrición de postlarvas se han incrementado en los últimos años (Kawamura \& Takami 1995, Kawamura et al. 1998, Roberts et al. 1999, Searcy-Bernal et al. 2001, Gordon et al. 2006), éstos no serían suficientes para conocer como afecta la composición de las biopelículas de diatomeas y su madurez sobre el crecimiento y supervivencia de las postlarvas, resultando esta técnica de cultivo una práctica variable y poco predecible, siendo más frecuente cuando la obtención de biopelículas de diatomeas para la fijación de postlarvas, se realiza de manera empírica, mediante la inmersión de los sustratos de fijación en agua de mar cruda. Esta práctica impide realizar una selección específica de las diatomeas debido a la variación estacional de éstas en el agua de mar, en términos de cobertura y de diversidad de especies, con la subsecuente variabilidad de las tasas de crecimiento y supervivencia postlarval (Searcy-Bernal et al. 1992). De este modo, la optimización de la producción de semillas de abalón a nivel comercial, pasaría por un apropiado control de la fuente alimenticia inicial como ha sido descrito por Seki \& Kan-no (1977), Seki (1980) y Hahn (1989), respecto de las especies de diatomeas (Seki 1980, Kawamura et al. 1998); cobertura celular, eficiencia de digestión, ingestabilidad (Takami et al. 1997, Kawamura et al. 1998, Daume et al. 2000, Roberts 2001), composición bioquímica (Dunstan et al. 1996) y valor nutricional
(Brown et al. 1996, 1997, Renaud et al. 1999). Otra característica importante para optimizar la oferta alimentaria sobre semillas de abalón, sería la facilidad con la cual éstas son removidas e ingeridas desde los sustratos de fijación, principalmente, debido a que las postlarvas de abalón pastorean en forma selectiva ciertas especies de diatomeas, según sean las características de tamaño celular, fuerza de adhesión y forma de crecimiento (Mathews \& Cook 1995, Kawamura \& Takami 1995, Kawamura et al. 1998).

Este estudio propone generar conocimiento respecto de la utilización de una biopelícula microalgal, compuesta por una mezcla de diatomeas bentónicas dominantes previamente aisladas, desde estanques de fijación de postlarvas de abalón, con el objetivo de optimizar el asentamiento y crecimiento de postlarvas de abalón Haliotis rufescens (Swainson, 1822) a través del preacondicionamiento del sustrato de fijación, por medio de la utilización de una biopelícula mixta específica de diatomeas bentónicas.

\section{Material y métodos}

Estimación de las curvas de crecimiento para las

Se cultivaron cepas de diatomeas marinas bentónicas previamente aisladas (Tabla 1) en cultivos axénicos e identificadas preliminarmente como NV (Amphora $c f$. capitellata), NC (Nitzschia ovalis arnott), CC (Cylindrotheca closterium) y NAV (Navicula cryptocephala). Estas fueron cultivadas en el medio f/2 (Guillard 1975) suplementado con silicato de sodio $\left(\mathrm{Na}_{2} \mathrm{SiO}_{3}\right)$, con el objeto de estimar las curvas de crecimiento para cada una de las cepas seleccionadas. Posteriormente, los inóculos provenientes de cultivos primarios, fueron dispuestos bajo condiciones asépticas por medio de la utilización de una campana de flujo laminar, sobre cámaras multipozos (Corning, USA) estériles en un volumen final de $10 \mathrm{~mL}$, hasta alcanzar

Tabla 1

Diatomeas bentónicas aisladas para el desarrollo de biopelículas multiespecíficas

Benthic diatoms isolated for multispecific biofilm development

\begin{tabular}{lllcc}
\hline \multirow{2}{*}{ Especie } & \multirow{2}{*}{ Abreviación } & & \multicolumn{2}{c}{ Tamaño celular } \\
\cline { 4 - 5 } & & & Longitud $(\mu \mathrm{m})$ & Ancho $(\mu \mathrm{m})$ \\
\hline Amphora cf. capitellata & $\mathrm{NV}$ & Estanque de biologización & 8 & 4 \\
Nitzschia ovalis arnott & $\mathrm{NC}$ & Estanque reservorio & 14 & 6 \\
Cylindrotheca closterium & $\mathrm{CC}$ & Estanque de asentamiento & 30 & 4 \\
Navicula cryptocephala & $\mathrm{NAV}$ & Estanque reservorio & 12 & 7 \\
\hline
\end{tabular}


una concentración final de $1 \times 10^{4}$ células $\mathrm{mL}^{-1}$, en triplicado. Una vez inoculadas cada una de las cepas, los cultivos se incubaron hasta alcanzar la fase estacionaria de cultivo a $20 \pm 1^{\circ} \mathrm{C}$, con agitación manual dos veces por día y luz continua, mediante la utilización de una lámpara fluorescente de luz blanca de $36 \mathrm{~W}$. Los recuentos fueron realizados diariamente mediante la utilización de un microscopio invertido (Olympus ${ }^{\circledR}$ BX 51) por contraste de fase. La estimación de la cobertura celular se realizó mediante el recuento de al menos 1000 células.

\section{Estimación del efecto de la intensidad lumínica sobre el crecimiento de diatomeas bentónicas}

Con el objetivo de optimizar las condiciones de cultivo de microalgas bentónicas, se realizaron ensayos tendientes a evaluar el efecto de la intensidad de la luz sobre cada una de las cepas utilizadas en el estudio. Para este propósito, se utilizó un túnel de gradiente lumínico, donde la fuente luminosa se ubicó en uno de los extremos del túnel en función de la distancia con respecto de la lámpara. Las intensidades lumínicas experimentales fueron de 10,50 y $100 \mu \mathrm{mol} \mathrm{m}^{-2} \mathrm{~s}^{-1}$, las cuales fueron estimadas, con un cuantómetro marca LI-COR Inc. (LI250A Light Meter). Previo a la evaluación de las curvas de crecimiento, cada una de las cepas fue aclimatada, por al menos tres generaciones, de acuerdo a la condición de intensidad lumínica a evaluar. Una vez que los cultivos fueron aclimatados, se estimó el crecimiento de las diatomeas bajo las condiciones experimentales por medio del recuento en triplicado, a partir de cada uno de los inóculos de las distintas diatomeas bentónicas estudiadas. Las condiciones de cultivo y el recuento celular se realizaron de acuerdo a la metodología descrita en el apartado anterior.

\section{Estimación de las tasas de crecimiento de los cultivos de diatomeas bentónicas}

Las tasas de crecimiento para las distintas cepas de diatomeas seleccionadas fueron evaluadas por medio de la ecuación de Guillard (Stein 1979), la cual describe la tasa media de duplicación de las células de acuerdo a la siguiente ecuación:

$$
\mathrm{K}=\left[3,322 /\left(t_{2}-t_{1}\right)\right] \times\left[\log \left(N_{2} / N_{1}\right)\right]
$$

donde: $K$ representa la tasa de crecimiento específico de la microalga, expresada como duplicación celular por día, $N_{1}$, densidad celular al comienzo del experimento, $N_{2}$, densidad celular al final del experimento, $t_{1}$, tiempo inicial para el cultivo experimental, $t_{2}$, tiempo final para el cultivo experimental.

\section{Biologización masiva de placas de fijación larval en "raceways"}

Con el objeto de promover la biologización de las diatomeas bentónicas seleccionadas sobre placas de fijación para ensayos masivos en "raceways", los cultivos masivos individuales en botellones de policarbonato de $20 \mathrm{~L}$ de capacidad, fueron cosechados desde fase preestacionaria mediante decantación y concentradas hasta alcanzar el número de células necesarias para inocular un volumen de $5 \mathrm{~m}^{3}$ a una concentración final de diatomeas de $1 \times 10^{4}$ células $\mathrm{mL}^{-1}$.

Una vez obtenida la biomasa algal de las distintas especies de diatomeas, y previo a la inoculación de la mezcla de diatomeas bentónicas, dos "raceways" de 5 $\mathrm{m}^{3}$ de volumen fueron llenados con agua de mar filtrada a $1 \mu \mathrm{m}$. Posteriormente fue desinfectada mediante cloro a una concentración de $25 \mathrm{mg} \mathrm{L}^{-1}$ por al menos $12 \mathrm{~h}$. Transcurrido este tiempo, se neutralizó el cloro activo con tiosulfato de sodio a una concentración de $1 \mathrm{M}$, más aireación vigorosa del agua. Inactivado el cloro desde el agua de los "raceways", se inocularon los nutrientes a partir de fertilizantes agrícolas (grado técnico) como urea para el nitrógeno $\left(0,15 \mathrm{~g} \mathrm{~L}^{-1} ; 60 \%\right.$ de $\left.\mathrm{N}\right)$, superfosfato triplex para el fósforo $\left(0,03 \mathrm{~g} \mathrm{~L}^{-1} ; 48 \% \mathrm{P}\right)$ y silicato de sodio $\left(0,013 \mathrm{~g} \mathrm{~L}^{-1}\right)$. Una vez fertilizados los "raceways", se inocularon las distintas especies de diatomeas bentónicas (Amphora cf. capitellata, Nitzschia ovalis arnott, Cylindrotheca closterium y Navicula cryptocephala) para obtener un mezcla homogénea a una concentración final $1 \times 10^{4}$ células $\mathrm{mL}^{-1}$. Posterior a la inoculación y para mejorar la homogenización y con ello la adherencia de las diatomeas sobre las placas de fijación larval, se restauró la aireación por 5 minutos con las placas de asentamiento en posición paralela al fondo de los estanques. Transcurrido este tiempo, se suspendió el suministro de aire por 30 minutos. Finalmente, los contenedores con las placas de fijación fueron colocados de manera perpendicular al fondo del estanque y dejados sin aireación por 12 horas, para luego reponer el suministro de aire por 7 días consecutivos bajo condiciones controladas; esto último, para promover la formación de las biopelículas de diatomeas bentónicas. Como control, se utilizó un estanque fertilizado de acuerdo a la metodología descrita anteriormente con agua de mar no filtrada, para promover el crecimiento de diatomeas bentónicas provenientes del agua de mar cruda. La cuantificación del crecimiento de células en el tratamiento con la adición de las diatomeas seleccionadas como en el control, se realizó diariamente de acuerdo a la metodología ya descrita, por medio de la extracción aleatoria de placas de fijación a partir de cada unos de los estanques experimentales. La comparación de los 
datos registrados para cobertura entre el tratamiento control y la mezcla de diatomeas bentónicas utilizadas en el tiempo, fue comparada estadísticamente mediante la prueba de ANCOVA con el programa STATISTICA 5.0. (StatSoft, Inc).

\section{Evaluación de la biopelícula microalgal multiespecífica sobre el asentamiento y crecimiento de postlarvas de abalón a nivel masivo}

Para conocer el efecto de la biopelícula de diatomeas bentónicas a nivel masivo, sobre el asentamiento y crecimiento de postlarvas de abalón, fueron evaluadas tres cohortes larvales a través de tres experimentos a nivel comercial, en empresas localizadas en la comuna de Caldera $\left(27^{\circ} 04^{\prime} 02,80^{\prime \prime} \mathrm{S} ; 70^{\circ} 49^{\prime} 20,22^{\prime \prime} \mathrm{W}\right)$, norte de Chile. Para cada experimento se dispusieron dos "raceways", de los cuales a cada uno se les incorporaron 20 contenedores con 25 placas de fijación, tanto para el tratamiento experimental (mezcla multiespecífica de diatomeas) como para el tratamiento control (biologización con agua de mar no filtrada). Una vez dispuestos los contenedores se inocularon larvas saludables de abalón de manera volumétrica a una densidad final de 1200 larvas. El recuento de semillas se monitoreó transcurridos 60 días para dos cohortes larvales y 120 días para otra cohorte larval, para evaluar el porcentaje de asentamiento y crecimiento postlarval. Para ello se retiró al azar el 15-20\% de las placas de fijación desde cada tratamiento como valor de proximidad al error, para luego cuantificar y estimar el número y la talla de las postlarvas asentadas, respectivamente. Finalmente, los datos recuperados fueron registrados en una planilla y analizados mediante una ANOVA de una vía y posteriormente un análisis múltiple de medias con el programa STATISTICA 5.0. (StatSoft, Inc)

\section{Análisis estadísticos}

Para evaluar en primera instancia la normalidad y la homogeneidad de las varianzas de los datos, los análisis de Bartlet \& Harley y Cochran fueron aplicados, respectivamente, de acuerdo a la metodología descrita por Underwood (1997). Debido a que los requisitos de normalidad y de homogeneidad de varianzas fueron cumplidos, los datos no fueron transformados (Sokal \& Rohlf 1995, Underwood 1997). Análisis de ANCOVA (para comparación de pendientes) y ANOVA de una vía, fueron luego aplicados con $P<0,05$. Análisis a posteriori fueron realizados por medio de la utilización de StudentNewman-Keuls (Steel \& Torie 1988, Underwood 1997) con una significancia de $P<0,05$.

\section{Resultados}

\section{Evaluación del crecimiento de diatomeas bentónicas}

Las curvas de crecimiento muestran que las cepas $\mathrm{NC} \mathrm{y}$ CC alcanzaron la fase pre-estacionaria a partir de las 144 horas de cultivo, respecto de las diatomeas NV y NAV, las cuales alcanzaron su fase pre-estacionaria de crecimiento a las 72 y 168 h, respectivamente (Fig. 1).

Los resultados tendientes a evaluar el efecto de la intensidad lumínica sobre las distintas diatomeas seleccionadas a 10,50 y $100 \mu \mathrm{mol} \mathrm{m}^{-2} \mathrm{~s}^{-1}$, reveló que las distintas cepas alcanzaron los mejores crecimientos en términos de cobertura a la intensidad de $50 \mu \mathrm{mol} \mathrm{m}^{-2} \mathrm{~s}^{-1}$, a las $120 \mathrm{~h}$ de cultivo para las cepas NC, CC y NV y 144 h para la cepa NAV (Fig. 2). La estimación de la velocidad media de duplicación de cada cepa, dada por el valor $\mathrm{K}$ de cada uno de los cultivos, mostró también que las mayores magnitudes de este índice fueron obtenidas cuando los cultivos microalgales fueron expuestos a 50 $\mu \mathrm{mol} \mathrm{m} \mathrm{m}^{-2} \mathrm{~s}^{-1}$. Esto último se evidencia al observar el incremento en tasa de duplicación a medida que aumenta la intensidad de luz sobre los cultivos, donde las magnitudes del valor $\mathrm{K}$ tienden a alcanzar un máximo de 1,156 a una intensidad lumínica de $50 \mu \mathrm{mol} \mathrm{m}^{-2} \mathrm{~s}^{-1}$ para

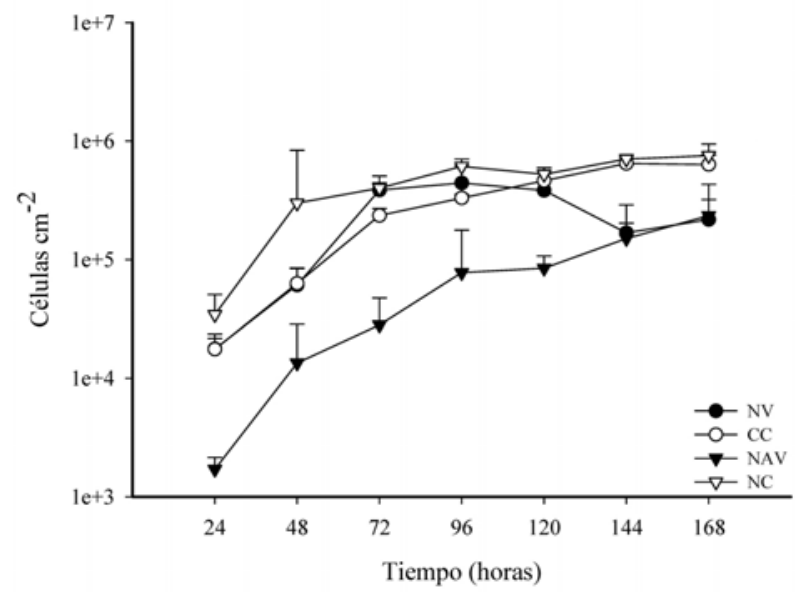

Figura 1

Curvas de crecimiento de las diatomeas aisladas: NV, Amphora cf. capitellata; CC, Cylindrotheca closterium; NAV, Navicula cryptocephala y NC, Nitzschia ovalis arnott. Los datos representan el promedio de muestras en triplicado \pm desviación estándar (DS)

Cell growth of isolated diatoms: NV, Amphora cf. capitellata; CC, Cylindrotheca closterium; NAV, Navicula cryptocephala and NC, Nitzschia ovalis arnott. Data represent the average of three samples \pm standard deviation (SD) 


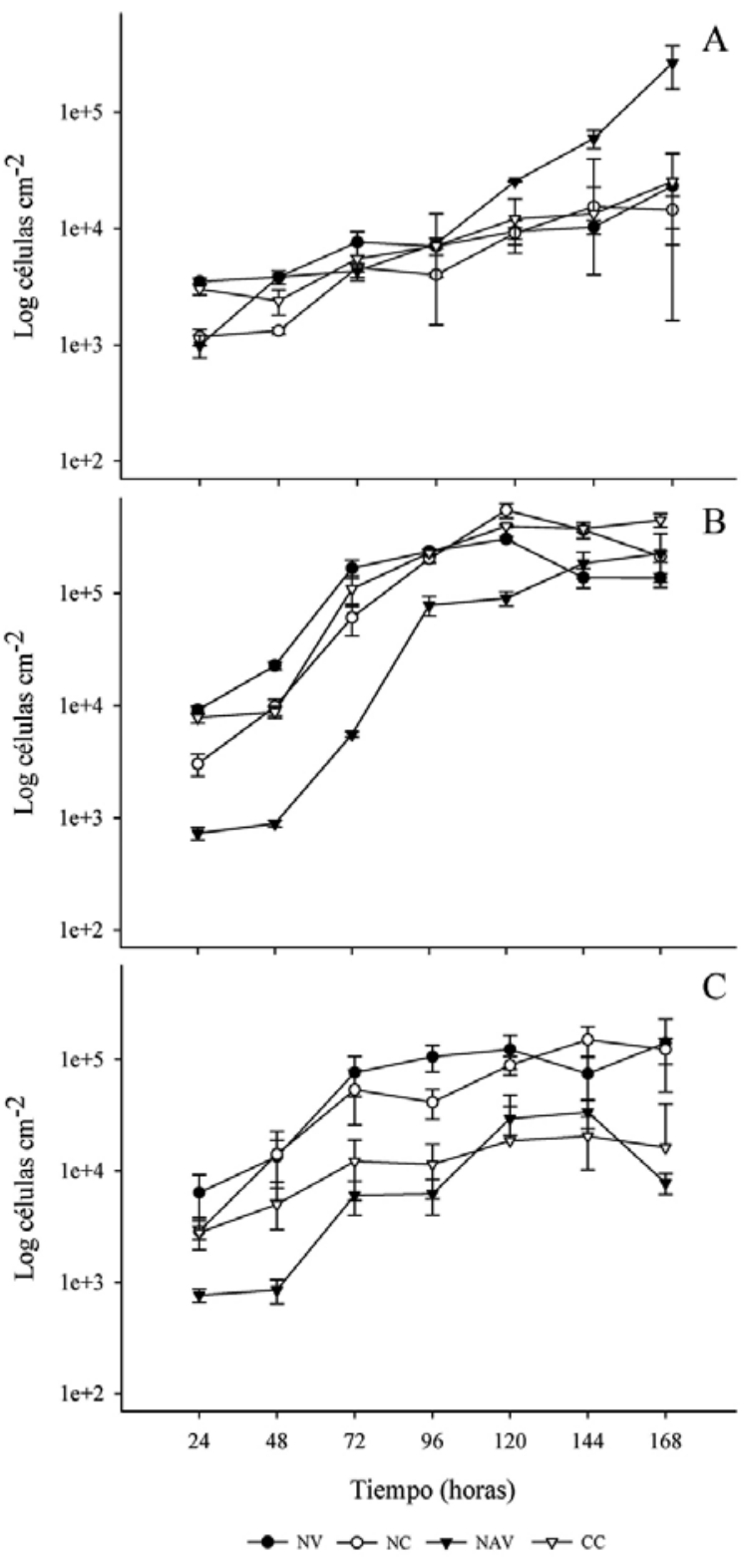

Figura 2

Evaluación del efecto de la intensidad lumínica sobre el crecimiento de monocultivos de NV, NC, NAV y CC expuestos a: A) $10 \mu \mathrm{mol} \mathrm{m} \mathrm{m}^{-2} \mathrm{~s}^{-1}$, B) $50 \mu \mathrm{mol} \mathrm{m}^{-2} \mathrm{~s}^{-1}$

у C) $100 \mu \mathrm{mol} \mathrm{m} \mathrm{m}^{-2} \mathrm{~s}^{-1}$. Los datos representan el promedio de muestras en triplicado \pm desviación estándar. Abreviaciones en Fig. 1

Evaluation of the light intensity effect on diatom growth from monocultures of $\mathrm{NV}, \mathrm{NC}, \mathrm{NAV}$ and $\mathrm{CC}$ exposed

to A) $10 \mu \mathrm{mol} \mathrm{m}^{-2} \mathrm{~s}^{-1}$, B) $50 \mu \mathrm{mol} \mathrm{m} \mathrm{m}^{-2} \mathrm{~s}^{-1}$ and

C) $100 \mu \mathrm{mol} \mathrm{m} \mathrm{m}^{-2} \mathrm{~s}^{-1}$. Data represent the average of three samples \pm standard deviation.

Abbreviations in Fig. 1
Tabla 2

Valor $K$ de las cuatro especies de diatomeas bentónicas a diferentes intensidades lumínicas

$\mathrm{K}$ value of four benthic diatoms species at different light intensities

\begin{tabular}{lcccc}
\hline Especie & $\begin{array}{c}10 \\
\text { K-valor }\end{array}$ & $\begin{array}{c}50 \\
\text { K-valor }\end{array}$ & $\begin{array}{c}100 \\
\text { K-valor }\end{array}$ & $P$-valor(*) \\
\hline NV & 0,0173 & 0,986 & 0,545 & 0,0002 \\
NC & 0,1334 & 1,156 & 0,653 & 0,0006 \\
NAV & 0,6750 & 0,693 & 0,293 & 0,0179 \\
CC & 0,1910 & 0,784 & 0,173 & 0,0009 \\
\hline * P-valor $<0,05$ indica diferencias estadisticas &
\end{tabular}

la cepa $\mathrm{NC}$, a diferencia del valor mínimo con una magnitud de 0,0173 para $10 \mu \mathrm{mol} \mathrm{m}^{-2} \mathrm{~s}^{-1}$ para la cepa $\mathrm{NV}$ (Tabla 2). El análisis estadístico de los resultados mediante ANOVA evidenció diferencias significativas entre las medias de los valores $\mathrm{K}$ para las diferentes intensidades de luz $(P<0,05)$ (Tabla 2$)$.

\section{Biologización masiva de placas de fijación larval en "raceways"}

La biologización de placas de fijación larval para cada ensayo masivo realizado, mostró que bajo el protocolo desarrollado y las condiciones dadas, la biologización específica con la mezcla de diatomeas bentónicas desplegó mayor cobertura celular en comparación al control con agua de mar cruda (ANCOVA, $\mathrm{F}_{(1,102)}=54,92$; $P<0,001)$, ambos tratamientos con la adición de fertilizantes agrícolas (Fig. 3). Estas diferencias de cobertura celular, entre ambos tratamientos, se observaron a partir de las 96 h de biologización, alcanzando éstas una diferencia de un orden de magnitud a las $168 \mathrm{~h}$ para el tratamiento con la mezcla específica de diatomeas, respecto del control (Fig. 3A). El recuento desglosado de cada una de las especies de diatomeas durante la formación de la biopelícula multiespecífica, evidenció una proporcionalidad muy homogénea en la cobertura de las distintas especies (Fig. 3B).

\section{Evaluación de la biopelícula microalgal multiespecífica sobre el asentamiento y el crecimiento de postlarvas de abalón a nivel masivo}

La evaluación realizada de la mezcla de diatomeas bentónicas sobre el asentamiento y crecimiento de postlarvas de abalón a nivel masivo, fue a los 60 y 120 días posteriores a la fijación larval. 


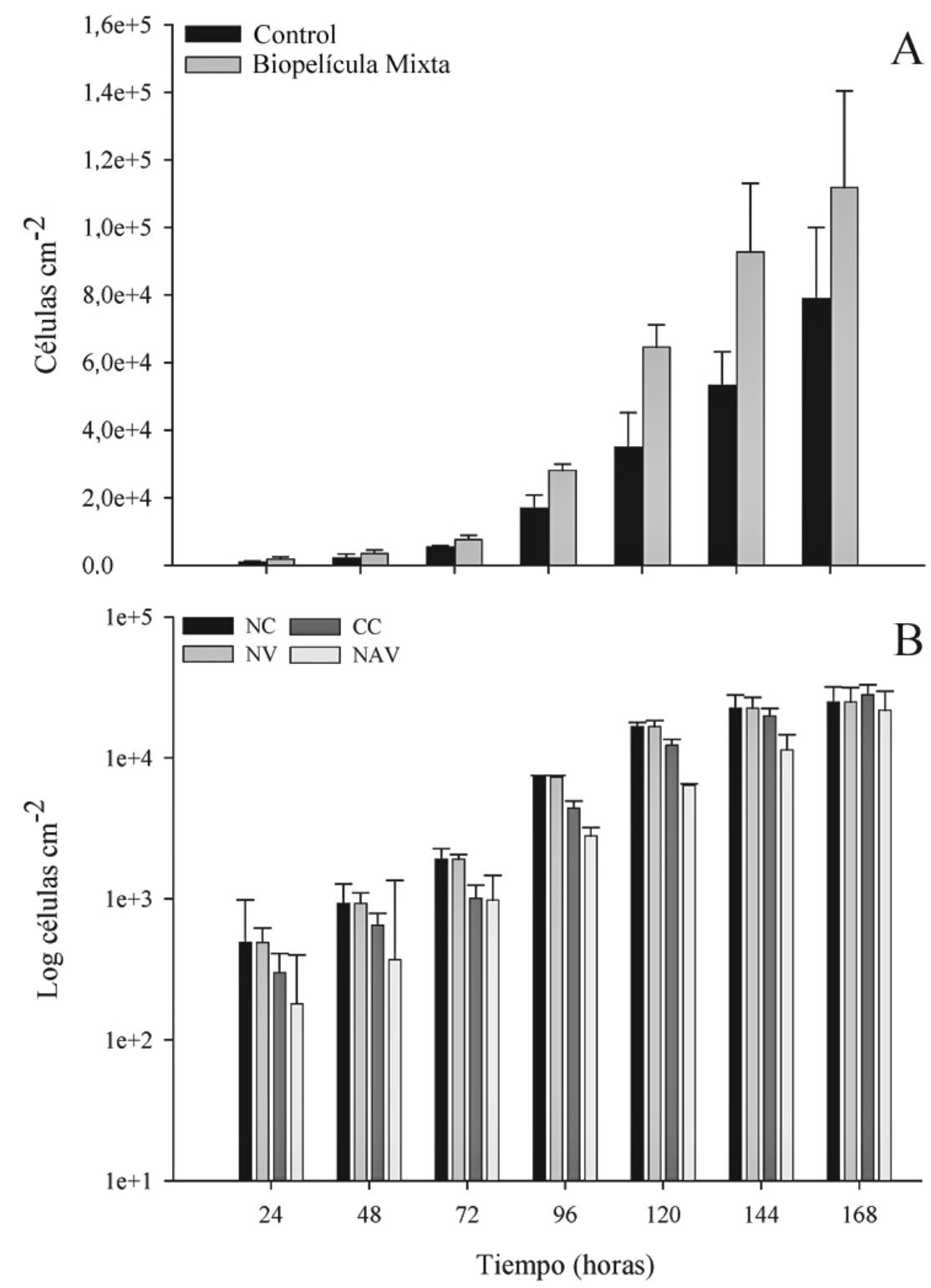

Figura 3

A) Variación de la cobertura celular en placas de fijación de larvas de Haliotis sp. a distintas horas después de la inoculación de una mezcla multiespecífica de diatomeas bentónicas respecto del control (agua de mar sin suplemento de diatomeas).

B) Recuento de diatomeas NC, NV, CC y NAV durante la formación de una biopelícula multiespecífica. Los resultados representan el promedio de tres experimentos masivos \pm desviación estándar.

Abreviaciones en Fig. 1

A) Variation of cell coverage on larval setting plates of Haliotis sp. at different hours after the inoculation of a multispecific mixture of benthic diatoms respect to the control (seawater without diatom supplement). B) Count of NC, NV, CC and NAV during a multispecific biofilm formation. Results represent the average of three massive experiments \pm standard deviation.

Abbreviations in Fig. 1 
La Fig. 4 muestra el número de semillas recolectada por $\mathrm{cm}^{2}$ y la talla de estas (en milímetros) a partir del análisis del 15-20\% de las placas de 120 días de fijación, tanto del tratamiento con la mezcla específica de diatomeas, como del control. Este ensayo muestra, que la utilización de la mezcla multiespecífica de diatomeas bentónicas mejoró el retorno de semillas en un 120\%, esto es, de un promedio de 3,65 $\pm 2,43$ semillas $\mathrm{cm}^{-2}$ a $8,13 \pm 6,22$ semillas $\mathrm{cm}^{-2}$ (ANOVA, $\mathrm{F}_{(1,94)}=20,69 ; P<$ $0,05)$. En relación a la talla de las semillas, transcurrido el mismo período la utilización de esta biopelícula microalgal, evidenció un incremento de 6,87 $\pm 1,96$ a $8,32 \pm 2,24 \mathrm{~mm}$ respecto del control (ANOVA, $\mathrm{F}_{(1,556)}=$ $53,32 ; P<0,05)$.
El análisis de dos cohortes larvales después de 60 días de efectuada la fijación, reveló que la utilización del criterio de una biologización selectiva mediante el uso de la biopelícula microalgal mejoró el retorno de semillas $\mathrm{cm}^{-2}$ de 20,26 $\pm 20,09$, respecto del control, que alcanzó promedios de 16,14 $\pm 15,06$ (ANOVA, $F_{(1,}$ $\left.{ }_{286)}=5,94 ; P<0,05\right)$. Al analizar el promedio de las tallas, se observó que aquellas semillas recuperadas mediante la utilización de la biopelícula microalgal $(6,34 \pm 3,33$ $\mathrm{mm})$ evidenciaron mejores crecimientos respecto del control $(4,46 \pm 2,68 \mathrm{~mm})$, siendo estas diferencias estadísticamente significativas (ANOVA, $\mathrm{F}_{(1,952)}=83,39$; $P<0,05)$ (Fig. 5).

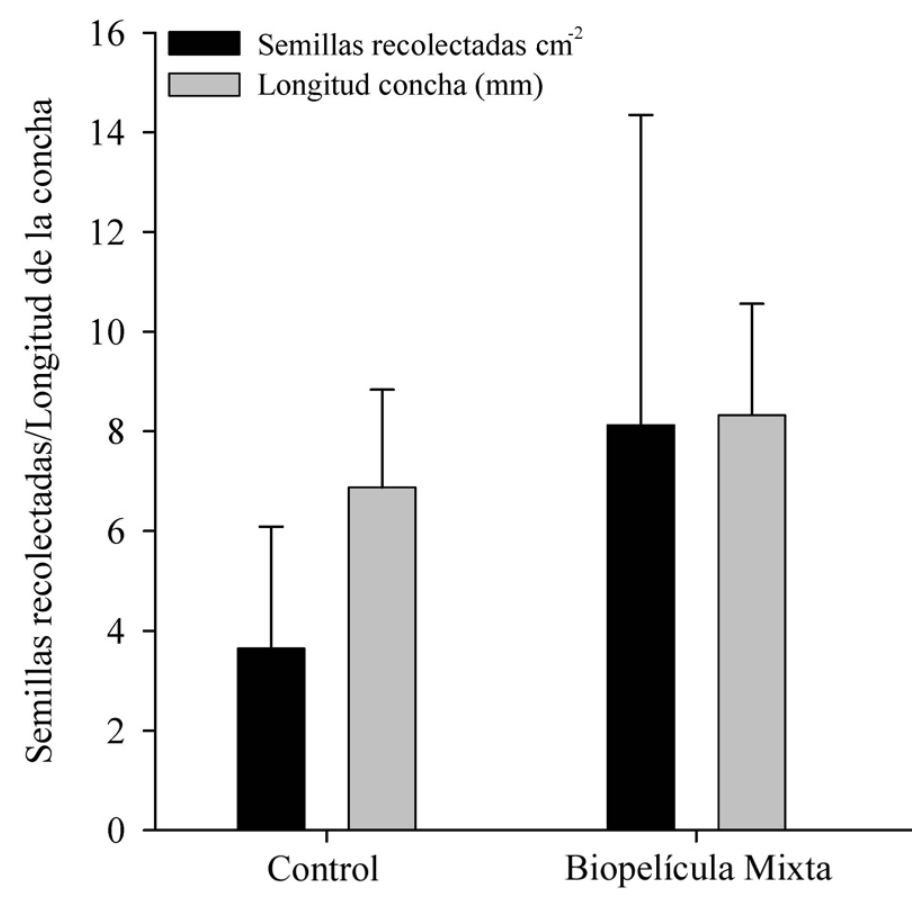

Tratamientos

Figura 4

Número de semillas recolectadas y longitud de la concha de postlarvas de abalón para una cohorte posterior a 120 días desde la fijación larval por medio de la utilización de una biopelícula mixta y de placas de asentamiento preacondicionadas con un flujo continuo de agua de mar (control). Los datos representan el promedio de muestras en triplicado aleatoriamente desde el estanque \pm desviación estándar

Number of collected seed and shell length of abalone postlarvae of a cohort after 120 days post settlement using diatom biofilm mixture and settlement plates preaconditionated under a continuous flow of seawater (control).

Data represent the average of three samples \pm standard deviation 


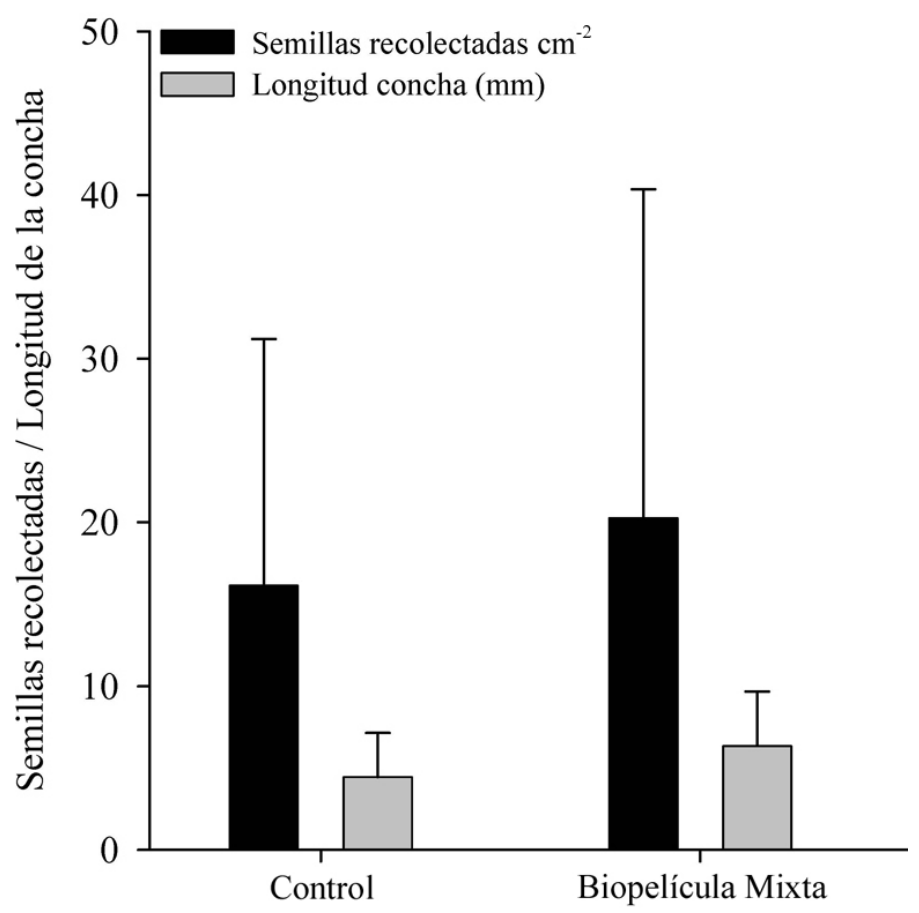

Tratamientos

Figura 5

Número de semillas recolectadas y longitud de la concha a partir del análisis de dos cohortes postlarvales posterior a 60 días desde la fijación por medio de la utilización de una biopelícula mixta y de placas de asentamiento preacondicionadas con un flujo continuo de agua de mar cruda (control). Los datos representan el promedio de muestras en triplicado aleatoriamente desde el estanque \pm desviación estándar

Number of collected seed and shell length of two abalone postlarvae cohorts after 60 days post settlement using diatom biofilm mixture and settlement plates preaconditionated under a continuous flow of raw seawater (control).

Data represent the average of three samples \pm standard deviation

\section{Discusión}

Utilizar criterios de biologización selectiva para la formación de biopelículas de diatomeas bentónicas de manera específica, constituye una práctica simple y eficiente en comparación a la biologización inespecífica. Esta última, principalmente tiene como inconveniente la variabilidad estacional en la abundancia de las especies de diatomeas bentónicas, sin garantizar una cobertura apropiada que sostenga el crecimiento de las postlarvas. El suministro apropiado de diatomeas durante los primeros meses de cultivo, se constituye como la principal fuente de alimento para organismos recién asentados (Kawamura \& Kikuchi 1992, Daume et al. 1997, Kawamura et al. 1998, Searcy-Bernal et al. 2001, Gordon et al. 2006), hasta alcanzar alrededor de los primeros 7 $8 \mathrm{~mm}$ de longitud.
Los resultados de la evaluación del crecimiento de las diatomeas seleccionadas en este estudio bajo una intensidad de $50 \mu \mathrm{mol} \mathrm{m} \mathrm{m}^{-2} \mathrm{~s}^{-1}$, mostraron una máxima producción celular por sobre las $10^{5}$ células $\mathrm{cm}^{-2}$ transcurridas $120 \mathrm{~h}$ para las cepas NC, CC y NV y $144 \mathrm{~h}$ para la cepa NAV (Fig. 2). Como ha sido descrito por Pulz \& Scheibenbogen (1998) y Avendaño-Herrera \& Riquelme (2007), el principal factor que controla la productividad de las diatomeas es la intensidad lumínica. En nuestro estudio, la reducción en la densidad celular de los cultivos estaría afectada a intensidades por debajo de $10 \mu \mathrm{mol} \mathrm{m} \mathrm{m}^{-2} \mathrm{~s}^{-1}$ y por encima de $50 \mu \mathrm{mol} \mathrm{m} \mathrm{m}^{-2} \mathrm{~s}^{-1}$, que coinciden con los estudios realizados por Tuji (2000) y Avendaño-Herrera \& Riquelme (2007). Irradiaciones por sobre el umbral óptimo han sido descritas como fotoinhibición, lo cual provocaría una caída en el proceso de fotosíntesis, que en algunos casos podría provocar la 
muerte de los cultivos (Tuji 2000), siendo este fenómeno dependiente de la naturaleza de la especie (Castenholz 1964). Similares resultados han sido descritos para el cultivo de Nitzschia americana, la cual se fotoinhibe con intensidades en el rango de las 74-140 $\mu_{\mathrm{mol} \mathrm{m}}^{-2} \mathrm{~s}^{-1}$ (Miller $\&$ Kamykowski 1986). De acuerdo a nuestros resultados, $50 \mu \mathrm{m} \mathrm{m}^{-2} \mathrm{~s}^{-1}$ de luz constante y la utilización de $\mathrm{f} / 2$ como medio de cultivo, permite producir masivamente las especies seleccionadas para su inoculación a nivel comercial en "raceways", con la finalidad de dirigir la oferta alimentaria de manera específica para mejorar el asentamiento y crecimiento de postlarvas de abalón. En este estudio se obtuvo una alta cobertura en términos de células $\mathrm{cm}^{-2}$, para la mezcla multiespecífica, a partir de, las diatomeas seleccionadas, transcurridas $168 \mathrm{~h}$, en comparación del control sin la inoculación de biopelículas microalgales a partir de cultivos masivos (Fig. 3). Particularmente, la evaluación de la cobertura celular para cada una de las cuatro cepas seleccionadas, revela que éstas se adhirieron a las placas de fijación en una proporción bastante homogénea. Esto aseguraría la disponibilidad de las distintas diatomeas inoculadas como suministro alimenticio para postlarvas de abalón, con la subsecuente ventaja en el asentamiento y crecimiento (Figs. 4 y 5), como ya ha sido descrito por Kawamura \& Kikuchi (1992) y Daume et al. (1997). Esto último, tendría mayor importancia debido a que estas biopelículas estarían creciendo sobre las placas de fijación de manera uniforme constituyendo una fuente diversa de alimento en un período de tiempo relativamente corto, en comparación a la biologización inespecífica (tratamiento control). Cabe mencionar que en algunos casos, esta práctica comienza meses antes del desove de los abalones, dependiendo de las condiciones de cultivo (e.g. luz, agua, temperatura, etc.), por lo que la disponibilidad de alimento en cuanto a cantidad y diversidad de especies varía temporalmente entre cohortes larvales.

Recientemente, se ha demostrado que las mezclas de diatomeas proporcionan mejores tasas de supervivencia y mayor crecimiento que los cultivos unialgales de diatomeas bentónicas (Gordon et al. 2004, 2006). Esto podría estar relacionado, a que una dieta multiespecífica entregaría mejores aportes nutricionales respecto de una monoespecífica (Kawamura et al. 1998), o también a un efecto sinérgico entre las diatomeas respecto de sus interacciones (Epifanio 1979). Sin embargo, y aunque el diseño experimental no permite establecer el efecto monoespecífico de estas cepas, podría ocurrir que en el tratamiento control, las biopelículas que también son multiespecíficas, podrían contener poblaciones que tuviesen un efecto adverso sobre el asentamiento y crecimiento de semillas de abalón. Respecto al último punto, se ha reportado que la utilización de Navicula cf. laevis como dieta monoespecífica en el cultivo de post larvas de Haliotis discus hannai; si bien estimula el asentamiento (Gordon et al. 2004) no proporciona buenos resultados como única dieta para el crecimiento temprano de postlarvas (Gordon et al. 2006), lo que incluso ha sido reportado como una consecuencia de la secreción de sustancias que podrían acumularse en forma gradual sobre las placas de fijación (Wen \& Chen 2002). Así mismo, Cocconeis scutellum var parva (Takami et al. 1997) es una especie de diatomea bentónica que proporciona un estímulo en el asentamiento con bajo efecto sobre el crecimiento de postlarvas de Haliotis discus hannai.

Estos antecedentes podrían explicar los mejores resultados desplegados por los ensayos analizados transcurridos 60 y 120 días posteriores al asentamiento larval (Figs. 4 y 5). Estos muestran diferencias significativas sobre el asentamiento y crecimiento de postlarvas posterior a 120 días del asentamiento con un aumento en el retorno de semillas del $120 \%(8,31 \pm 6,22$ semillas $\mathrm{cm}^{-2}$ de placa de fijación) en comparación a la biologización inespecífica (control), con una tasa de retorno de 3,65 $\pm 2,43$ semillas $\mathrm{cm}^{-2}$ (Fig. 4). Consideramos que estas evidencias estarían relacionadas con la sinergia entre las distintas diatomeas utilizadas, y la naturaleza de los exopolisacáridos de la biopelícula con la subsecuente mejora del valor nutricional. También se observó que la utilización de nuestra biopelícula microalgal mejoró el crecimiento larval (Figs. 4 y 5) de semillas recolectadas después de 60 y 120 días posteriores a la fijación larval.

Diversos factores pueden influir en el valor nutricional de los cultivos de diatomeas, entre los cuales destacan, el tipo de polisacáridos extracelulares (EPS) generado por las diatomeas, el estado fisiológico del cultivo, la microflora asociada (Kawamura 1996), dentro de la cual destaca la presencia de bacterias. Sin embargo, nuevos estudios debieran abordar el comportamiento de la biopelícula multiespecífica, cuando es predada por las postlarvas de abalón, para conocer cómo es la sucesión de las diatomeas que componen la biopelícula respecto de las comunidades fitoplantónicas y bacterianas presentes en el agua de cultivo.

\section{Agradecimientos}

Este estudio fue financiado por el Fondo de Fomento al Desarrollo Científico y Tecnológico (FONDEF, CONICYT) Grant N ${ }^{\circ}$ D02I-1097. Los autores agradecen al Dr. Marco Ortiz por su colaboración en el análisis estadístico de los resultados, a las empresas de cultivos 
Vinycon Chilena Ltda. y Pesquera Camanchaca por la buena disposición en la utilización de su infraestructura como también en la cooperación del seguimiento de los ensayos y la colaboración anónima de los evaluadores a través de cada uno de los comentarios para enriquecer el presente estudio.

\section{Literatura citada}

Avendaño-Herrera R \& C Riquelme. 2007. Production of a diatom-bacteria biofilm in a photobioreactor for aquaculture applications. Aquacultural Engineering 36: 97-104.

Brown M, G Dunstan, S Norwood \& K Miller. 1996. Effects of harvested stage and light on the biochemical composition of the diatom Thalassiosira pseudonana. Journal of Phycology 32: 64-73.

Brown M, S Jeffrey, J Volkman \& G Dunstan. 1997. Nutritional properties of microalgae for mariculture. Aquaculture 151: 315-331.

Bryan P \& P Qian. 1998. Induction of larval attachment and metamorphosis in the abalone Haliotis diversicolor (Reeve). Journal of Experimental Marine Biology and Ecology 223: 39-51.

Castenholz R. 1964. The effect of daylength and light intensity on the growth of littoral marine diatoms in culture. Physiologia Plantarum 17: 951-963.

Daume S, S Brand \& W Woelkerling. 1997. Effects of postlarval abalone (Haliotis rubra) grazing on the epiphytic diatom assemblage of coralline red algae. Molluscan Research 18: 119-130.

Daume S, A Krsinich, S Farrell \& M Gervis. 2000. Settlement early growth and survival of Haliotis rubra in response to different algal species. Journal of Applied Phycology 12: 479-488.

Dunstan G, H Baillie, S Barett, \& J Volkmann. 1996. Effect of diet on the lipid composition of wild and cultured abalone. Aquaculture 140: 115-127.

Epifanio C. 1979. Growth in bivalve mollusks: nutritional effects of two or more species of algae diets fed to the American oyster Crassostrea viriginica (Gmelin) and the hard clam Mercenaria mercenaria (L.). Aquaculture 18: 112 .

Gordon N, M Shpigel, S Harpaz, J Lee \& A Neori. 2004. The settlement of abalone (Haliotis discus hannai Ino) larvae on culture layers of different diatoms. Journal of Shellfish Research 23: 561-568.

Gordon N, A Neori, M Shpigel, J Lee \& S Harpaz. 2006. Effect of diatom diets on growth and survival of the abalone Haliotis discus hannai postlarvae. Aquaculture 252: 225233.

Guillard R. 1975. Culture of phytoplankton for feeding marine invertebrates, 29 pp. Plenum Press, New York.
Hahn K. 1989. Nutrition and growth of abalone. En: Hahn $\mathrm{KO}$ (ed). Handbook of culture of abalone and other marine gastropods, pp. 135-156. CRC Press, Boca Raton.

Kawamura T. 1996. The role of benthic diatoms in the early life stage of the Japanese abalone Haliotis discus hannai. En: Watanabe Y, Y Yamashita \& Y Oozeki (eds). Survival strategies in early life stages of marine resources, pp. 335367. Balkema, Rotterdam.

Kawamura T \& S Kikuchi. 1992. Effects of benthic diatoms on settlement and metamorphosis of abalone larvae. Suisazoshoku 40: 403-409. [In Japanese with English Abstract].

Kawamura T \& H Takami. 1995. Analysis of feeding and growth rate of newly metamorphosed abalone Haliotis discus hannai fed on four species of benthic diatom. Fisheries Science 61: 357-358.

Kawamura T, R Roberts \& C Nicholson. 1998. Factors affecting the food value of diatoms strains for post larval abalone Haliotis iris. Aquaculture 160: 81-88.

Matthews I \& P Cook. 1995. Diatom diet of abalone postlarvae (Haliotis midae) and the effect of pre-grazing the diatom overstorey. Marine and Freshwater Research 46: 545-548.

Miller R \& D Kamykowski. 1986. Effect of temperature, alkalinity, salinity, irradiance and diurnal periodicity on growth and photosynthesis in the diatom Nitzschia americana: light-satured growth. Journal of Phycology 22: 339-348.

Pulz O \& K Scheibenbogen. 1998. Photobioreactors: design and performance with respect to light energy input. Advances in Biochemical Engineering/Biotechnology 59: 123-152.

Renaud S, L van Thinh \& D Parry. 1999. The gross chemical composition and fatty acids composition of 18 species of tropical Australian microalgae for possible use in mariculture. Aquaculture 170: 147-159.

Roberts R. 2001. A review of settlement cues for larval abalone (Haliotis sp.). Journal of Shellfish Research 20(2): 571586.

Roberts R, T Kawamura \& C Nicholson. 1999. Growth and survival of postlarvae abalone (Haliotis iris) in relation to development and diatom diet. Journal of Shellfish Research 18: $243-250$.

Searcy-Bernal R, A Salas-Garza, R Flores-Aguilar \& $\mathbf{P}$ Hinojosa-Rivera. 1992. Simultaneous comparison of methods for settlement and metamorphosis induction in the red abalone (Haliotis rufescence). Aquaculture 105: 241250.

Searcy-Bernal R, L Velez-Espino \& C Anguiano-Beltran. 2001. Effect of biofilm density on grazing and growth rates of Haliotis fulgens postlarvae. Journal of Shellfish Research 20: $587-591$. 
Seki T. 1980. An advanced biological engineering system for abalone seed production. En: International Symposium on Coastal Pacific Marine Life, Western Washington University. 1980, Bellingham, pp. 45-54.

Seki T \& H Kan-no. 1977. Synchronized control of early life in the abalone, Haliotis discus hannai Ino. Haliotidae, Gastropoda. Bulletin of the Tohoku Regional Fisheries Research Laboratory 38: 143-153. [In Japanese with English Abstract]

Sokal R \& F Rohlf. 1995. Biometry, 878 pp. Freeman and Company, San Francisco.

Stein J. 1979. Handbook of phycological methods, culture and growth measurements, 446 pp. Cambridge University Press, Cambridge.

Steel R \& H Torrie. 1988. Bioestadística: principios y procedimientos, $662 \mathrm{pp}$. McGraw-Hill, México.
Takami H, T Kawamura \& Y Yamashita. 1997. Survival and growth rates of post-larval abalone Haliotis discus hannai fed conspecific trail mucus and/or benthic diatom Cocconeis scutellum var. Parva. Aquaculture 152: 129-138.

Tuji A. 2000. The effect of irradiance on the growth of different of freshwater diatoms: implications for succession in attached diatoms communities. Journal of Phycology 36: 659-661.

Underwood A. 1997. Experiments in ecology: their logical design and interpretation using analysis of variance, 504 pp. Cambridge University Press, Cambridge.

Viana M. 2006. ¿Qué pasa con la acuacultura de abulón en Chile y México? Boletín Informativo Trimestral de la Sociedad Mexicana de Abulón 3(8): 1-7.

Wen $\mathbf{Z} \& \mathbf{Y}$ Chen. 2002. Perfusion culture of the diatom Nitzschia laevis for ultra-high yield of eicosapentanoic acid. Process Biochemistry 38: 523-529.

Recibido el 08 de abril de 2009 y aceptado el 20 de noviembre de 2009 